\title{
Ionic Liquid Facilitated Recovery of Metals and Oxygen from Regolith
}

\author{
Laurel J. Karr, ${ }^{1}$ Peter A. Curreri, ${ }^{2}$ Gary S. Thornton, ${ }^{3}$ Kevin E. Depew, ${ }^{4}$ John M. Vankeuren, ${ }^{5}$ Matthew Regelman, ${ }^{6}$ \\ and Eric T. Fox ${ }^{7}$ \\ NASA/Marshall Space Flight Center, Huntsville, AL, 35812, USA \\ Matthew J. Marone ${ }^{8}$ \\ Mercer University, Macon, GA, 31207, USA \\ David N. Donovan ${ }^{9}$ \\ Qualis Corporation, Huntsville, AL, 35806, USA \\ and \\ Mark S. Paley ${ }^{10}$ \\ AZ Technology, Huntsville, AL, 35806, USA
}

\section{Introduction}

Regolith, being largely composed of metal oxides, has the potential to be an excellent in situ source of metals, such as iron, nickel and aluminum, as well as oxygen. There is, however, no current technology that is suited for the in space separation of metal oxides into their constituent elements. Terrestrial methods require large volumes/masses of hazardous reagents. Alternative methods, such as molten oxide electrolysis, have been investigated, but these processes generally require high temperatures to operate. Such a requirement imposes significant material compatibility restraints, necessitates high energy inputs, and may pose a threat to the health and safety of the crew.

This paper discusses a novel process that uses ionic liquids (ILs) to recover high purity metals and oxygen from regolith and meteorite materials. ILs are organic salts that are molten at or near room temperature, and can have a number of attractive properties including wide liquidus ranges and high thermal and electrochemical stability. The structure of an IL can be readily modified to tune these properties, allowing the synthesis of task specific ILs. Together, these attributes give ILs great utility in a number of space related areas, such environmental control and life support, in space repair and manufacture, and in situ resource utilization.

The IL-based process is composed of three steps. In the first step, an acidic IL is used to dissolve the metal oxides (Eq. 1). The second step electrolyzes water to produce hydrogen for use in the third step and oxygen for use as breathing air or propellant. The third step consists of the concurrent recovery of the dissolved metals via electrodeposition and regeneration of the spent IL using hydrogen flowing over a platinum black electrode (Eq. 2).

$$
\begin{gathered}
2 \mathrm{HSO}_{4}^{-}+\mathrm{MO} \rightarrow 2 \mathrm{SO}_{4}^{2-}+\mathrm{M}^{2+}+\mathrm{H}_{2} \mathrm{O} \\
\mathrm{M}^{2+}+2 \mathrm{e}^{-} \rightarrow \mathrm{M} \| 2 \mathrm{SO}_{4}^{2-}+\mathrm{H}_{2} \rightarrow 2 \mathrm{HSO}_{4}^{-}+2 \mathrm{e}^{-}
\end{gathered}
$$

\footnotetext{
${ }^{1}$ Scientist Emeritus, EM22 Material Test, Chemistry \& Contamination Control Branch.

${ }^{2}$ Scientist, EM31 Materials Science and Metallurgy Branch.

${ }^{3}$ Mechanical Engineer, ES63 Instrument Development Branch.

${ }^{4}$ Project Manager, HP30 ISS Projects Office

${ }^{5}$ Electrical Engineer, ES61 Flight Systems Integration \& Test Branch.

${ }^{6}$ Summer Intern, EM31 Materials Science and Metallurgy Branch.

${ }^{7}$ Ionic Liquid Science Lead, EM22 Material Test, Chemistry \& Contamination Control Branch.

${ }^{8}$ Associate Professor, Department of Physics.

${ }^{9}$ Technician, EM22 Material Test, Chemistry \& Contamination Control Branch.

${ }^{10}$ Chemist, EM22 Material Test, Chemistry \& Contamination Control Branch.
} 
The regenerated IL can then be recycled to restart the first step of the process. The IL used in this process is 100\% recovered, so a process using this approach would not require a supply of reagents other than the initial volume.

Regolith and meteorites are composed of a variety of elements, such as iron, nickel, silicon, and aluminum. During the electrodeposition step, the application of sequential steps of increasing reducing potential allows the recovery of each element in a high purity state. The high electrochemical stability of ILs also allows for the recovery of metals that are reduced at potentials that fall outside the electrochemical windows of many solvents.

\section{Results}

Initial testing of the sequential electroplating of metals was conducted using a $0.1 \mathrm{M}$ solution of 1-ethyl-3methylimidazolium hydrogen sulfate (EMI $\mathrm{HSO}_{4}$ ) and an equimolar amount of metals in water. The addition of water both reduced the amount of IL required for the experiment and increased the mass transfer kinetics in solution. The dissolved metals had a composition typical of nickel iron meteorites (93\% iron, 7\% nickel by mass). Cyclic voltammetry conducted on these solutions using a glassy carbon working electrode showed that the presence of metals resulted in the formation of two new, convoluted reduction peaks (Fig. 1). The reduction of nickel is responsible for the peak near $-0.8 \mathrm{~V}$ and the reduction of iron(II) is responsible for the peak near $-1.1 \mathrm{~V}$. This peak assignment was verified by cyclic voltammetry performed on solutions containing a single metal.

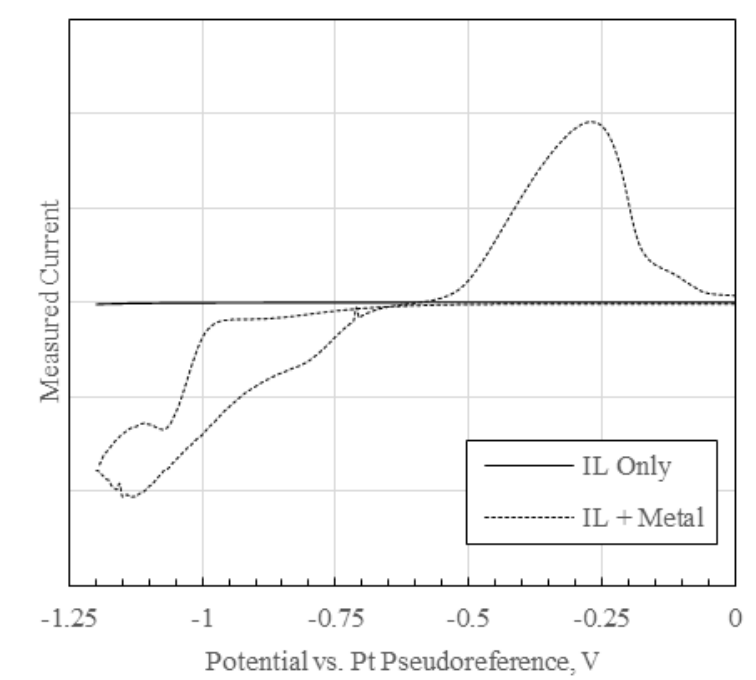

Fig. 1 Cyclic voltammograms of $0.1 \mathrm{M}$ EMI $\mathrm{HSO}_{4}$ in water with (IL + metal) and without (IL only) dissolved metals.

Electrodeposition from a bulk solution was performed at $-0.95 \mathrm{~V}$ and $-1.30 \mathrm{~V}$ to reduce nickel and iron respectively. At $-0.95 \mathrm{~V}$ almost no current was measured $(\mathrm{i}<0.1 \mathrm{~mA}$ ) and after 14 hours of run time, the electrode had no visible metal deposits. SEM EDS analysis on the electrode surface confirmed that no metal reduction occurred. At $-1.30 \mathrm{~V}$ the initial current was approximately $1 \mathrm{~mA}$, but this value began to increase rapidly after 20 minutes (Fig. 2). This change corresponded with the formation of metal deposits on the surface of working electrode (Fig. 3). This current continued to increase rapidly until the one hour point where the electrode was fully covered with metal. The noise in the chronoamperogram is the result of changes in the electrode surface area as metal deposited onto and flaked off of the surface. During the course of the experiment, significant quantities of metal flakes collected on the cell floor. The electrodeposition was run for approximately 6 hours, and was terminated when the temperature of the electrolyte began a rapid increase in temperature. At this time, based on the total charge passed through the working electrode, approximately half of the dissolved metal was plated out of solution. The deposited metal had a composition of $93 \%$ iron and 7\% nickel, as quantified by EDS SEM. This is identical to the composition of the material used to prepare the electrolyte solution, indicating that the process did not preferentially reduce one of the component species. 


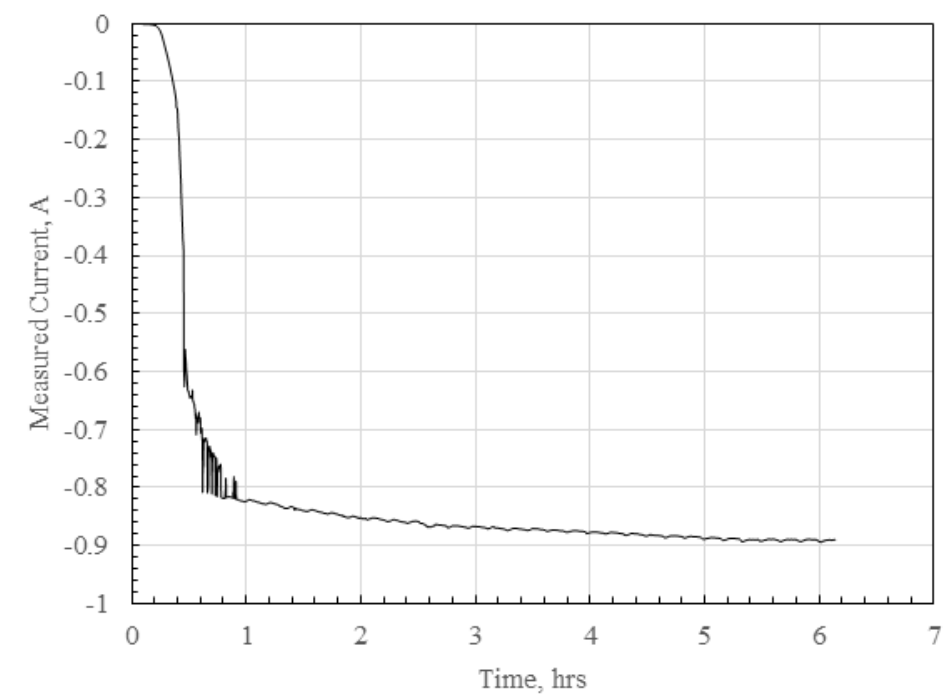

Fig. 2 Chronoampergoram of of a solution of $0.1 \mathrm{M} \mathrm{EMI} \mathrm{HSO}_{4}$ and $0.1 \mathrm{M} \mathrm{Fe} / \mathrm{Ni}$ in water with an applied potential of $-1.3 \mathrm{~V} \mathrm{vs}$. a Pt pseudoreference.
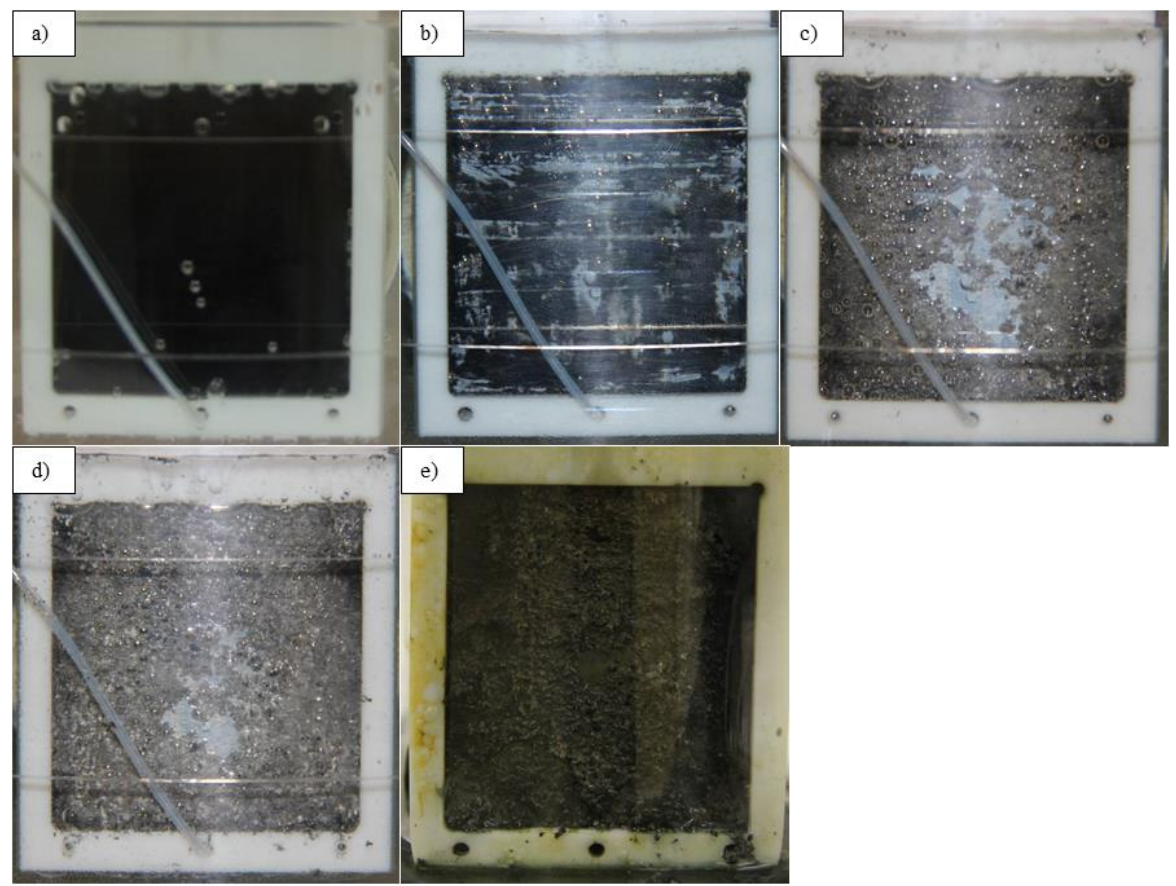

Fig. 3 The glassy carbon working electrode after a) $0 \mathrm{~min}$, b) $25 \mathrm{~min}$, c) $35 \mathrm{~min}$, d) $55 \mathrm{~min}$, and e) $375 \mathrm{~min}$ of electrodeposition in a $0.1 \mathrm{M}$ solution of $\mathrm{EMI} \mathrm{HSO}_{4}$ and metal in water with an applied potential of $-1.30 \mathrm{~V}$ vs. a Pt pseudoreference.

\section{Conclusions}

A process using ILs to recover metal from an aqueous solution containing an acidic IL was successfully demonstrated at room temperature. Additionally, the IL reagent was successfully regenerated, showing that the process is well suited for use in long duration exploration missions when the resupply of reagents is not an option. Further refinement of the electrochemical regime and apparatus is required and should allow for the recovery of high purity metal suitable for use as feedstock. 